\title{
Implante imediato associado a enxerto xenógeno e provisionalização imediata em área infectada: relato de caso
}

Immediate implant associated with a xenogen graft and immediate provisionalization in an infected area: clinical case Implante inmediato asociado con injerto de xenógeno y provisionalización inmediata en área infectada: reporte de caso Verônica Porto Ramos SAMPAIO' Diego Filipe Bezerra SILVA ${ }^{2}$

Flávio Marcel Pereira BARREIRO 3 Hiarles Barreto Sampaio BRITO ${ }^{3}$

Francisco Juliherme Pires de ANDRADE ${ }^{4}$

Daliana Queiroga de Castro GOMES ${ }^{5}$

Instituto de Odontologia da Paraíba, Pós-graduada em Implantodontia, Campina Grande, Paraíba, Brasil

${ }^{2}$ Universidade Estadual da Paraíba, Mestrando do Programa de Pós-Graduação em Odontologia, Campina Grande, Paraíba, Brasil

${ }^{3}$ Instituto de Odontologia da Paraíba, Professor Mestre do curso de Implantodontia, Campina Grande, Paraíba, Brasil

${ }^{4}$ Instituto de Odontologia da Paraíba, Professor Doutor, Campina Grande, Paraíba, Brasil

${ }^{5}$ Universidade Estadual da Paraíba, Professora Doutora do Programa de Pós-Graduação em Odontologia, Campina Grande, Paraíba, Brasil

\section{Resumo}

Introdução: A reabilitação com implantes dentários tornou-se possível de forma imediata a partir do conhecimento e aplicação da osseointegração. Esse procedimento propicia otimização entre o tempo de osseointegração e maturação óssea, além de minimizar a reabsorção alveolar, preservar a arquitetura gengival, e evitar novo procedimento cirúrgico. Objetivo: Evidenciar o sucesso de implante dentário unitário, instalado imediatamente após exodontia e curetagem de lesão periapical extensa, e a instalação da prótese com proservação de 33 meses. Relato do caso: Paciente do sexo feminino, 24 anos de idade, apresentou-se com uma parúlide na gengiva inserida acima do dente 25 e fratura coronal do mesmo. A tomografia computadorizada de feixe cônico (TCFC) revelou uma extensa área hipodensa na região do ápice da raiz do dente 25 , medindo 6,7 X 8,2 mm. A referida lesão já havia causado fenestração da parede vestibular, cerca de dois terços no sentido ápice-coroa. Foi instituído como plano de tratamento a instalação de implante dentário imediato com carga imediata. A prótese final foi instalada 13 meses após a instalação do implante. Decorridos 17 meses do procedimento cirúrgico, foi solicitada radiografia periapical de controle, onde foi possível avaliar trabeculado ósseo sadio em torno do implante. Conclusão: Tendo em vista o avanço dos biomateriais utilizados em reabilitação oral e o aprimoramento das técnicas e profissionais que a desempenham, conclui-se que é possível realizar implantes imediatos e reabilitação imediata em área infectada, mesmo em casos limítrofes, com segurança e efetividade, possibilitando a obtenção de resultado final satisfatório.

Descritores: Implantes Dentários; Infecção; Regeneração Óssea.

\section{Abstract}

Introduction: Rehabilitation with dental implants became possible immediately from the knowledge and application of osseointegration. This procedure provides optimization between osseointegration time and bone maturation, in addition to minimizing alveolar resorption, preserving the gingival architecture, and avoid a new surgical procedure. Objective: To demonstrate the success of a single dental implant, installed immediately after exodontia and curettage of extensive periapical lesion, and the installation of the prosthesis with 33 months of proservation. Case report: A 24-year-old female patient presented with a parsley in the gingiva inserted above the tooth 25 and coronal fracture of the same. Cone-beam computed tomography (CBCT) revealed an extensive hypodense area in the apex region of the tooth root 25 , measuring $6.7 \times 8.2 \mathrm{~mm}$. The said lesion had already caused fenestration of the vestibular wall, about two-thirds in the apex-crown direction. Installation of immediate dental implant with immediate loading was instituted as treatment plan. The final prosthesis was installed 13 months after implant installation. After 17 months of the surgical procedure, a periapical radiography of the control was requested, where it was possible to evaluate healthy bone trabeculation around the implant. Conclusion: With the advancement of biomaterials used in oral rehabilitation and the improvement of the techniques and professionals that perform it, it is concluded that it is possible to perform immediate implants and immediate rehabilitation in an infected area, even in borderline cases, with safety and effectiveness, obtaining a satisfactory final result.

Descriptors: Dental Implants; Infection; Bone Regeneration.

\section{Resumen}

Introducción: La rehabilitación con implantes dentales se hizo posible de inmediato gracias al conocimiento y la aplicación de la osteointegración. Este procedimiento proporciona optimización entre el tiempo de osteointegración y la maduración ósea, minimiza la resorción alveolar, preserva la arquitectura gingival y evita un nuevo procedimiento quirúrgico. Objetivo: Evidenciar el éxito de un único implante dental, instalado inmediatamente después de la extracción y legrado de una lesión periapical extensa, y la instalación de la prótesis con 33 meses de conservación. Reporte del caso: una paciente de 24 años de edad se presentó con una parulida en la encía insertada sobre el diente 25 y una fractura coronal. La tomografía computarizada de haz cónico (TCHC) reveló un área hipodensa extensa en la región del ápice de la raíz del diente 25, que mide 6,7 X 8,2 mm. Esta lesión ya había causado la fenestración de la pared vestibular, aproximadamente dos tercios en la dirección del ápice-corona. La implantación de un implante dental inmediato con carga inmediata se instituyó como un plan de tratamiento. La prótesis final se instaló 13 meses después de la instalación del implante. Después de 17 meses del procedimiento quirúrgico, se solicitó una radiografía de control periapical, en la que fue posible evaluar la trabeculación ósea sana alrededor del implante. Conclusión: en vista del avance de los biomateriales utilizados en la rehabilitación oral y la mejora de las técnicas y profesionales que lo realizan, se concluye que es posible realizar implantes inmediatos y rehabilitación inmediata en un área infectada, incluso en casos límite, de manera segura y efectiva. haciendo posible obtener un resultado final satisfactorio.

Descriptores: Implantes Dentales; Infección; Regeneración Ósea.

\section{INTRODUÇÃO}

A reabilitação com implantes dentários tornou-se possível de forma imediata a partir do conhecimento e aplicação da osseointegração, consolidada por Branemark ${ }^{1}$. Esse procedimento otimiza o tempo entre a osseointegração e maturação óssea, minimiza a reabsorção alveolar, preserva a arquitetura gengival e evita submeter o paciente a novo procedimento cirúrgico ${ }^{2,3}$. Branemark ${ }^{4}$ afirma que essa técnica tem apresentado resultados satisfatórios ao longo dos anos, assegurando sua execução, quando respeitadas as possíveis limitações que venham a existir.

Após uma exodontia é inevitável a reabsorção óssea que ocorre de forma acentuada nos 
maxilares, no decorrer dos $\operatorname{anos}^{5-7}$. Jensen et al. ${ }^{8}$ defendem que, apesar do sucesso da osseointegração, do aprimoramento das técnicas de enxertia, autógena ou não, e da biocompatibilidade dos biomateriais, que vêm sendo utilizados de forma rotineira nas reabilitações com implantes dentários, a remodelação óssea, seja ela a curto, médio ou longo prazo, não está livre de acontecer.

Como evidencia a literatura ${ }^{9,10}$, o advento dos implantes imediatos foi seguido pela necessidade da utilização da carga imediata. Segundo Carvalho et al. ${ }^{11}$, há controvérsias no que diz respeito à provisionalização imediata, seja com próteses adesivas ou fixas. No entanto, acredita-se que as mesmas podem auxiliar na preservação estrutural do tecido mole e proporcionam maior conforto póscirúrgico e estético ao paciente. Além disso, promovem condicionamento gengival, o que irá conduzir a estética final do caso ${ }^{12}$.

A utilização de biomateriais para regeneração óssea guiada (ROG), quando se faz opção pela reabilitação com implante imediato e há a presença de processo infeccioso crônico, é capaz de preencher gaps interalveolar e proporcionam uma barreira de proteção do sítio cirúrgico. Além disso, aumentam a previsibilidade do caso, bem como mantém a arquitetura gengival preservada, no intuito de fazer com que a estética final não seja prejudicada ${ }^{13}$.

Diante do exposto, o objetivo deste relato de caso clínico é evidenciar o sucesso de implante dentário unitário, instalado imediatamente após exodontia e curetagem de lesão periapical extensa, e a instalação da prótese com proservação de 33 meses.

\section{CASO CLÍNICO}

Paciente, 24 anos de idade, sexo feminino, branca, compareceu à clínica do Instituto de Odontologia da Paraíba (IOP) queixando-se de "inflamação na gengiva de um dente tratado com canal", com histórico de dor e edema local. Após realização de anamnese, foi possível constatar que a paciente não portava nenhuma doença sistêmica e não possuía, no momento do exame clínico, sintomatologia dolorosa. Ao exame físico intraoral, observou-se uma parúlide na gengiva inserida acima do dente 25 e fratura coronal do mesmo (Figura 1). $\mathrm{Na}$ análise radiográfica foi possível observar uma área radiolúcida, bem circunscrita, de aproximadamente 5,0 $\mathrm{mm}$ de diâmetro, localizada no ápice radicular do dente 25 , sugestiva de cisto ou granuloma periapical. A tomografia computadorizada de feixe cônico (TCFC) revelou uma extensa área hipodensa na região do ápice da raiz do dente 25 , medindo $6,7 \times 8,2 \mathrm{~mm}$. A referida lesão já havia causado fenestração da parede vestibular, cerca de dois terços no sentido ápice-coroa (Figura 2). Após elaboração do plano de tratamento, a paciente consentiu a realização da exodontia, instalação de implante dentário imediato e posterior reabilitação. Cerca de uma hora antes da cirurgia, a paciente administrou $1 \mathrm{~g}$ de Amoxicilina. A exodontia foi executada de forma minimamente traumática (Figura 3), no intuito de preservar as paredes ósseas alveolares remanescentes, bem como as papilas interdentais, almejando previsibilidade estética final favorável.

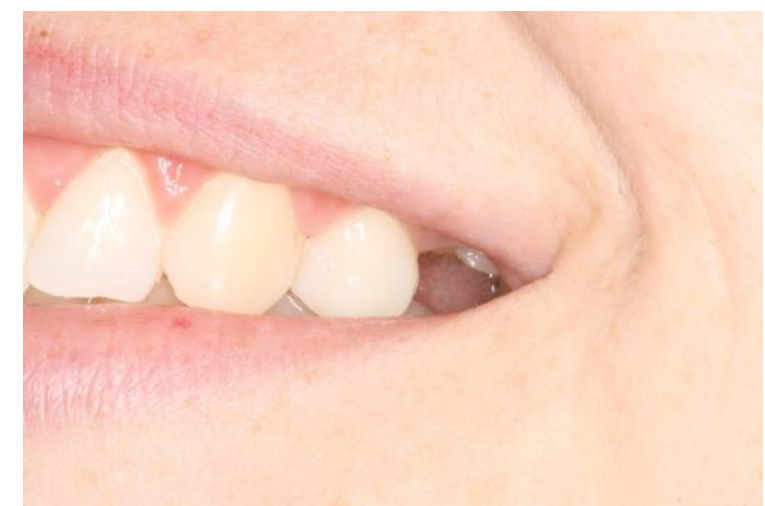

Figura 1. Imagem clínica inicial evidenciando fratura coronal do dente 15.

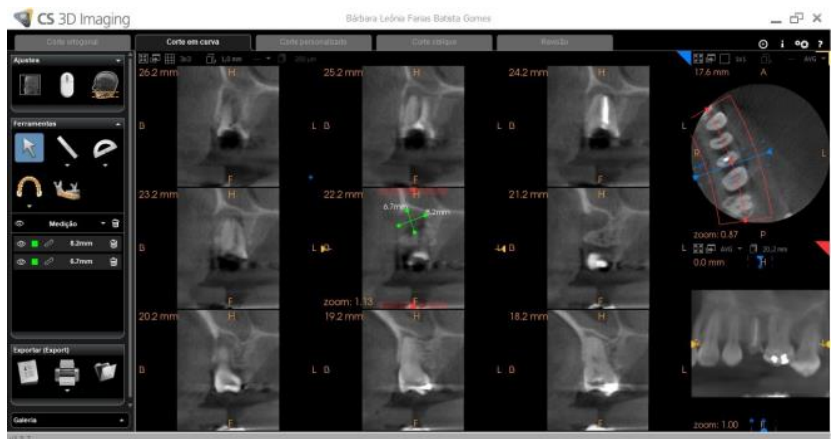

Figura 2. TCFC evidenciando extensa área hipodensa na região apical do dente 15 .

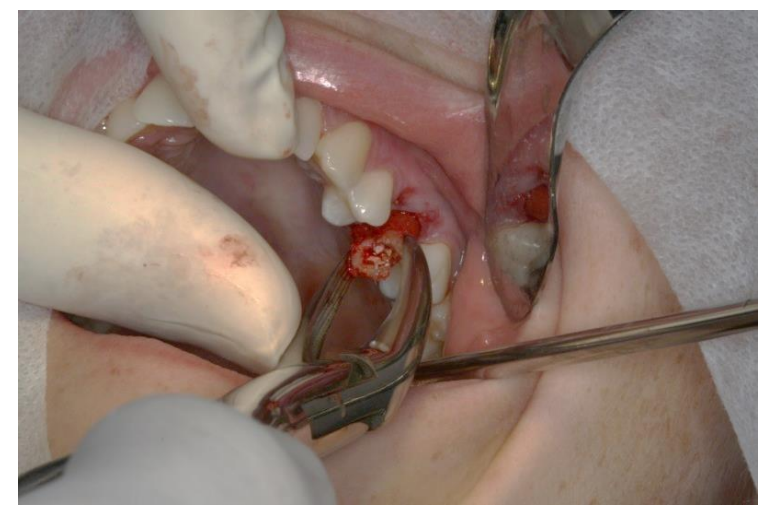

Figura 3. Exodontia minimamente traumática.

Após a exodontia, foi realizada anestesia local infiltrativa utilizando três tubetes de Mepivacaína 2\% (1:100.000 - Nova DFL), descolamento das papilas e incisões relaxantes nas regiões dos dentes 24 a 26, para confecção de retalho e exposição de toda área fenestrada (Figura 4) para curetagem da área infectada e posterior irrigação com soro fisiológico. Foi feita elevação de assoalho do seio maxilar utilizando elevador de Summers para melhor adaptação e travamento apical do implante (Alvim Cone Morse, 4,3 x 11,5mm, Neodent, Curitiba, PR, Brasil), que foi de $45 \mathrm{~N}$, preenchimento 
do gap com enxerto ósseo xenógeno de granulação fina (LUMINA-BONE POROUS, Critéria Biomateriais, São Paulo, SP, Brasil) e recobrimento por membrana de colágeno (LUMINA-COAT), Critéria Biomateriais, São Paulo, SP, Brasil) (Figuras 5 e 6).

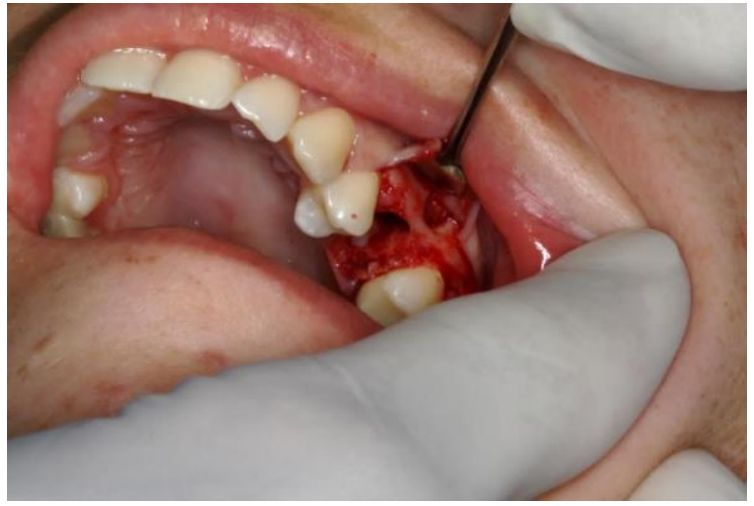

Figura 4: Exposição da área fenestrada.

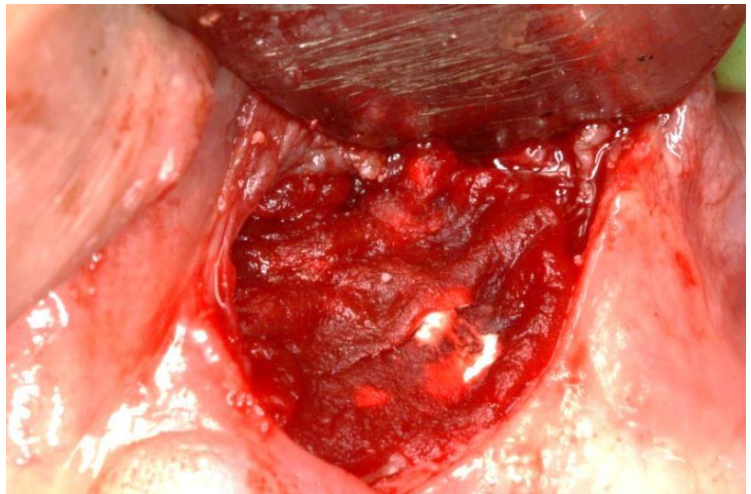

Figura 5: Preenchimento dos gaps e recobrimento com membrana de colágeno.

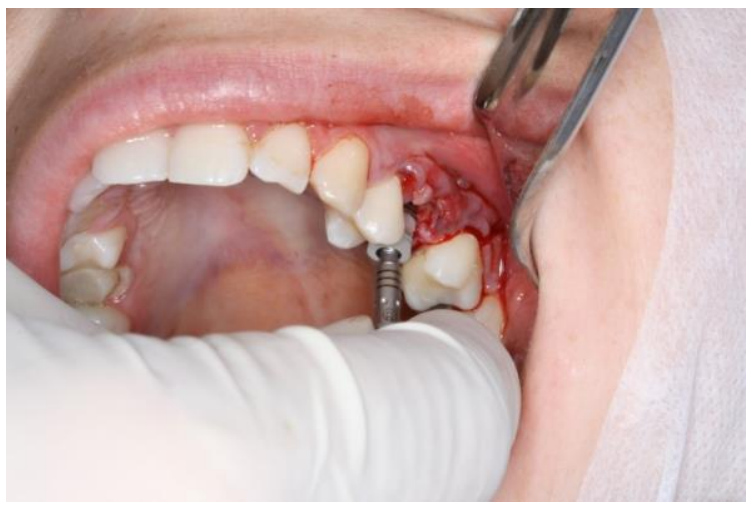

Figura 6: Implante sendo instalado no alvéolo.

Em seguida, o retalho foi reposicionado, suturado com fio de nylon 4.0, e confeccionada prótese provisória parafusada, junto ao componente intermediário protético, o qual foi selecionado por meio da altura do zênite gengival almejado, sobre o implante, porém, mantida em infraoclusão (Figura 7). Após a cirurgia, foi prescrito anti-inflamatório Nimesulida $100 \mathrm{mg}$ a cada 12 horas, durante três dias consecutivos; analgésico (Dipirona 500mg), em caso de dor, e antibiótico (Amoxicilina 500mg), a cada oito horas, durante sete dias consecutivos. Além disso, foram dadas devidas orientações pós-cirúrgicas verbalmente e por escrito.

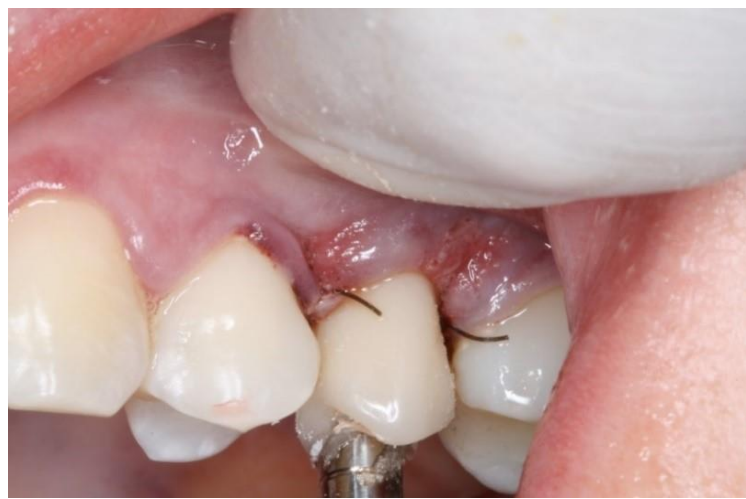

Figura 7: Sutura nas papilas e instalação de provisório imediato.

Após 30 dias da realização da cirurgia, foi solicitada nova TCFC (Figura 8) para avaliação comportamental do enxerto, tendo em vista a limitação do caso. Contudo, observou-se satisfatório início de osseointegração local bem como preservação das estruturas teciduais adjacentes e ausência de áreas de inflamação e/ou exsudato. A prótese final foi instalada 13 meses após a instalação do implante, pois, a paciente não retornou a clínica no período agendado. Decorridos 33 meses do procedimento cirúrgico, foi solicitada radiografia periapical de controle (Figura 9), onde foi possível avaliar trabeculado ósseo sadio em torno do implante.

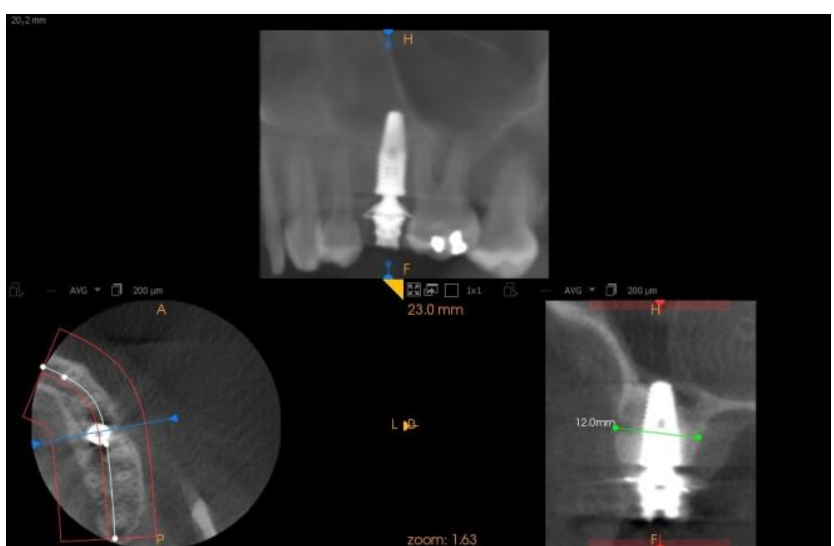

Figura 8: TCFC realizada 30 dias após realização do implante e enxerto alógeno particulado, mostrando o ganho de volume no sentido vestíbulopalatino.

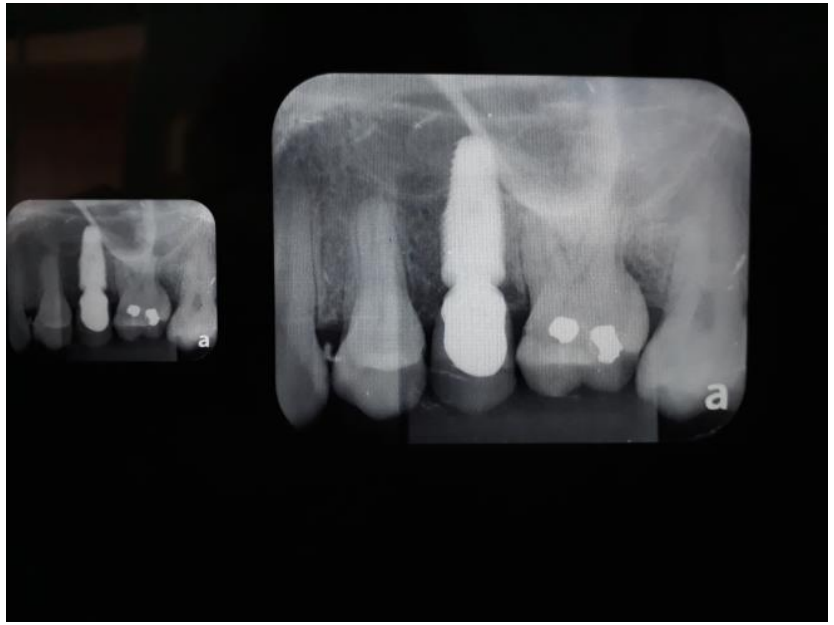

Figura 9: Radiografia periapical realizada 17 meses após colocação do implante. 


\section{DISCUSSÃO}

A instalação de prótese provisória imediatamente após a execução de implante imediato, mesmo na presença de processo infeccioso crônico local, na atualidade, é uma possibilidade para reabilitação ${ }^{14,15}$. Os biomateriais proporcionam reconstruções teciduais satisfatórias associadas à boa condição sistêmica do paciente, à técnica cirúrgica minuciosa, colaboração do paciente no pósoperatório, bem como ao comportamento biológico de cada indivíduo, oferecendo respaldo para o desempenho seguro dessa técnica ${ }^{14,15}$.

Branemark $^{16}$ defendia a reabilitação com implantes dentários em dois tempos cirúrgicos, assegurando que a osseointegração estivesse consolidada, a fim de evitar danos aos tecidos moles, o que acarretaria interferência na estética. Porém, estudos contemporâneos ${ }^{14,17}$ corroboram o presente relato de caso, evidenciando vantagens na colocação de implantes imediatos, com carga imediata e prótese mantida sem carga oclusal, o que proporciona preservação e manutenção da estrutura gengival.

No caso ora relatado, optou-se pela exodontia seguida de instalação de implante dentário, uma vez que o dente apresentava extenso comprometimento periapical e fenestração da tábua óssea vestibular. Ruskin et al. ${ }^{18}$ reforçam a efetividade da terapia realizada, assegurando a viabilidade e as vantagens de optar-se pela substituição de dentes comprometidos com fraturas, lesões periapicais ou tratados endodonticamente de maneira insatisfatória, por implantes dentários e, sempre que possível, instalação imediata da prótese. Por meio do acompanhamento de alguns casos de implantes imediatos e implantes convencionais, colocados após a cicatrização alveolar, Esposito et al. ${ }^{19}$ obtiveram melhor resultado final, inclusive, estético, bem como satisfação dos pacientes, nos implantes colocados logo após a exodontia, de acordo com o presente caso.

Penãrrocha et $\mathrm{al}^{3}{ }^{3}$ defenderam que o sucesso dos implantes imediatos depende de uma série de fatores, destacando a ausência de infecção, alvéolo preservado, bem como cortical vestibular satisfatória. Por outro lado, no presente caso foi possível obter êxito, por meio de adequado planejamento do caso, uma reabilitação adequada mesmo com a presença de processo infeccioso local e fenestração parcial da tábua óssea vestibular.

Buser et al. ${ }^{20}$ realizaram um estudo observacional e comprovaram a eficácia e o índice de sucesso em mais de $90 \%$ dos casos de implantes submetidos à carga imediata, quando os mesmos apresentavam estabilidade primária, o que possibilitou a execução de apenas um tempo cirúrgico. Dessa forma, confirma-se a vantagem de optar-se pela reabilitação com implantes imediatos e carga imediata, suprindo a necessidade estética do paciente.

De acordo com Sousa ${ }^{21}$, alguns estudos evidenciam a necessidade do preenchimento de gaps por biomateriais, após a instalação de implantes imediatos, com o objetivo de otimização dos resultados. O preenchimento de gaps com uso de enxertos ósseos particulados, mesmo não sendo autógenos, mostram-se eficazes na manutenção do alvéolo e dos tecidos moles adjacentes, proporcionando resultado estético favorável, o que foi verificado no caso ora relatado.

No que diz respeito à avaliação pós-cirúrgica de implantes submetidos à carga imediata em alvéolos pós-exodontia e locais já cicatrizados, Chaushu et al. ${ }^{22}$ observaram que, entre três e seis meses após a instalação da prótese, os implantes com carga imediata instalados em alvéolo pós-exodontia apresentaram risco de falha, diferentemente dos implantes submetidos à carga imediata em locais já cicatrizado que não apresentaram nenhum risco, dentro do mesmo período de tempo. Por outro lado, no presente caso, não houve nenhum tipo de falha com relação a técnica desenvolvida.

Em relação à reabsorção óssea marginal dos implantes, Lorenzoni et al. ${ }^{23}$ e Galli et al. ${ }^{24}$ evidenciaram que ocorre maior reabsorção quando a reabilitação é feita em dois estágios cirúrgicos, o que fortalece a escolha pela provisionalização imediata. Portanto, a utilização de implantes imediatos com carga imediata é uma técnica que pode ser indicada na prática clínica, desde que seja desempenhada com planejamento adequado e sejam tomadas as devidas precauções em cada caso.

\section{CONSIDERAÇÕES FINAIS}

Diante do caso exposto e tendo em vista o avanço dos biomateriais utilizados em reabilitação oral e o aprimoramento das técnicas e profissionais que a desempenham, conclui-se que é possível realizar implantes imediatos e reabilitação imediata em área infectada, mesmo em casos limítrofes, com segurança e efetividade, possibilitando a obtenção de resultado final satisfatório.

\section{REFERÊNCIAS}

1. Brånemark PI, Adell R, Breine U, Hansson BO, Lindström J, Ohlsson A. Intra-osseous anchorage of dental prostheses. I. Experimental studies. Scand J Plast Reconstr Surg. 1969;3(2):81-100.

2. Rosenquist B, Grenthe B. Immediate placement of implants into extraction sockets: implant survival. Int J Oral Maxillofac Implants. 1996;11(2):205-9.

3. Peñarrocha $M$, Uribe $R$, Balaguer J. Immediate implants after extraction. A review of the current situation. Med Oral. 2004;9(3):234-42.

4. Brånemark PI. Branemark Novum: protocolo para reabilitação bucal com carga imediata (same-day 
teeth): uma perspectiva global. São Paulo: Quintessence; 2001.

5. Carlsson GE, Bergman B, Hedegård B. Changes in contour of the maxillary alveolar process under immediate dentures. A longitudinal clinical and $\mathrm{x}$ ray cephalometric study covering 5 years. Acta Odontol Scand. 1967;25(1):45-75.

6. Novaes AB Jr, Vidigal Júnior GM, Novaes AB, Grisi MF, Polloni S, Rosa A. Immediate implants placed into infected sites: a histomorphometric study in dogs. Int $\mathbf{J}$ Oral Maxillofac Implants. 1998;13(3):422-7.

7. Misch CE. Implantes dentários contemporâneos. $2^{a}$ ed. São Paulo: Santos; 2000.

8. Jensen T, Schou S, Stavropoulos A, Terheyden H, Holmstrup P. Maxillary sinus floor augmentation with Bio-Oss or Bio-Oss mixed with autogenous bone as graft: a systematic review. Clin Oral Implants Res. 2012;23(3):263-73.

9. Müller A, Silva ACBR, Schimidt LTH. Implante unitário submetido à carga imediata. RGO (Porto Alegre). 2004;52(1):27-30.

10. de Oliveira AC, de Souza JR, Thomé G, Melo ACM, Sartori IAM. Implante imediato unitário em função imediata - relato de caso. RFO. 2008;13(1):70-4.

11. Carvalho PFM, Ciotti DL, Silva RC, Joly JC. Implantação e temporização imediata em áreas estéticas, sem abertura de retalho, utilizando implantes de diâmetro reduzido: Relato de caso clínico. Rev ImplantNews. 2008;5(8):307-12.

12. Ferreira LCP, Brito CR, Lehn CN, Siqueira JTT. Avaliação de implantes osseointegráveis submetidos à função imediata comparados à função tardia. Rev ImplantNews. 2010;7(1):13-20.

13. Tazima MFGS, Vicente YAMVA, Moriya T. Biologia da ferida e cicatrização. Medicina (Ribeirão Preto) 2008;41(3):259-64.

14. Thomé G, Borges AFS, Melo ACM, Bassi APF, Sartori IAM, Faot F. Implante imediato em local cronicamente infectado: avaliação após 12 meses. RGO (Porto Alegre). 2007;55(4):417-21.

15. Salmen FS, Oliveira MR, Gabrielli MAC, Piveta ACG, Pereira Filho VA, Gabrielli MFR. Enxerto ósseo para reconstrução óssea alveolar. Revisão de 166 casos. Rev Col Bras Cir. 2017;44(1):33-40.

16. Brånemark PI. Protesis tejido integradas: la osseointegración en la odontologia clínica. Berlim: Quintessence; 1987.

17. Nunes PA, Medeiros MM, Soder MV, Klein ALL, Hasse PN, Pfau EA. Implante e provisionalização imediata com resina composta em area estética. Arq Ciênc Saúde UNIPAR. 2014;18(1):65-8.

18. Ruskin JD, Morton D, Karayazgan B, Amir J. Failed root canals: the case for extraction and immediate implant placement. J Oral Maxillofac Surg. 2005;63(6):829-31.
19. Esposito M, Grusovin MG, Polyzos IP, Felice P, Worthington HV. Interventions for replacing missing teeth: dental implants in fresh extraction sockets (immediate, immediate-delayed and delayed implants). Cochrane Database Syst Rev. 2010;(9):CD005968.

20. Buser D, Mericske-Stern R, Bernard JP, Behneke A, Behneke N, Hirt HP, Belser UC, Lang NP. Long-term evaluation of non-submerged ITI implants. Part 1: 8-year life table analysis of a prospective multi-center study with 2359 implants. Clin Oral Implants Res. 1997;8(3):161-72.

21. Sousa MAF. Considerações relativas à colocação imediata de implantes em alvéolos pós-extração. Artigo de Revisão Bibliográfica Mestrado Integrado em Medicina Dentária. Faculdade de Medicina Dentária, Universidade do Porto, 2014.

22. Chaushu G, Chaushu S, Tzohar A, Dayan D. Immediate loading of single-tooth implants: immediate versus non-immediate implantation. A clinical report. Int $\mathrm{J}$ Oral Maxillofac Implants. 2001;16(2):267-72.

23. Lorenzoni M, Pertl C, Zhang K, Wimmer G, Wegscheider WA. Immediate loading of singletooth implants in the anterior maxilla. Preliminary results after one year. Clin Oral Implants Res. 2003;14(2):180-87.

24. Galli F, Capelli M, Zuffetti F, Testori T, Esposito M. Immediate non-occlusal vs. early loading of dental implants in partially edentulous patients: a multicentre randomized clinical trial. Peri-implant bone and soft-tissue levels. Clin Oral Implants Res. 2008;19(6):546-52.

\section{CONFLITO DE INTERESSES}

Os autores declaram não haver conflitos de interesse.

\section{AUTOR PARA CORRESPONDÊNCIA}

\section{Daliana Queiroga de Castro Gomes}

Departamento de Odontologia

Universidade Estadual da Paraíba, UEPB

Rua Baraúnas, 351, Bairro Universitário

58429-500 Campina Grande- PB, Brasil

Email: dqcgomes@hotmail.com 